\title{
Splenectomy as Part of Primary Cytoreductive Surgery for Advanced Ovarian Cancer A Retrospective Cohort Study
}

\author{
Ignacio Zapardiel, MD, PhD, Michele Peiretti, MD, PhD, Vanna Zanagnolo, MD, Roberto Biffi, MD, \\ Luca Bocciolone, MD, Fabio Landoni, MD, Giovanni Aletti, MD, Nicoletta Colombo, PhD, \\ and Angelo Maggioni, MD
}

\begin{abstract}
Background: The aim of surgical approach in advanced ovarian cancer should be the complete removal of all visible disease. Our purpose was to compare perioperative features and postoperative complications, and secondarily oncological outcomes, between patients who underwent splenectomy and those who did not at the time of surgery.

Materials and Methods: Thirty-three subjects underwent splenectomy, and we selected 99 controls with similar surgical characteristics but who did not undergo splenectomy. Data collected included perioperative details and follow-up data.

Results: Longer operating time (33 minutes longer; $P=0.02$ ), larger estimated blood loss $(812 \mathrm{~mL}$ more; $P=0.03)$, higher rate of intraoperative blood transfusions $(78.8 \%$ vs $42.4 \%$; $P<0.01)$, and intensive care unit stay $(1.4$ vs 0.5 days; $P<0.01)$ as well as higher pneumonia rate $(2 \%$ vs $0 \% ; P=0.01)$ were observed in the splenectomy group. Disease-free and overall survival rates were $30.3 \%$ and $66.6 \%$, respectively, in the splenectomy group, and $33.3 \%$ and $59.6 \%$, respectively, in the control group.

Conclusions: Splenectomy at the time of primary cytoreductive surgery for advanced ovarian cancer may contribute to achieve complete cytoreduction with low perioperative complication rate. This procedure seems to be an acceptable and rational intervention to increase the survival rates of those patients.
\end{abstract}

Key Words: Splenectomy, Ovarian cancer, Primary cytoreduction

Received October 31, 2011, and in revised form January 22, 2012.

Accepted for publication March 23, 2012.

(Int J Gynecol Cancer 2012;22: 968-973)

$\mathrm{O}$ varian cancer has the highest fatality-to-case ratio of all gynecologic cancers, as $62 \%$ of cases are diagnosed in advanced-stage disease; 21,880 new cases and 13,850 deaths are estimated during 2010 in the United States, which makes

Gynecologic Oncology Department, European Institute of Oncology, Milan, Italy.

Address correspondence and reprint requests to Ignacio Zapardiel

$\mathrm{MD}, \mathrm{PhD}$, Gynecologic Oncology Department, European

Institute of Oncology, Via Ripamonti 435. Milan, Italy.

E-mail: ignaciozapardiel@hotmail.com.

The authors declare that there are no conflicts of interest.

Copyright (C) 2012 by IGCS and ESGO

ISSN: $1048-891 \mathrm{X}$

DOI: $10.1097 /$ IGC.0b013e3182571479 this malignancy the fifth cancer related cause of death in females. $^{1}$

Standard approach of patients with advanced ovarian cancer should be primary cytoreduction followed by platinumbased chemotherapy. ${ }^{2-4}$ The aim of surgical approach should be the complete removal of all visible disease that would improve the oncological results. However, owing to technical reasons as well as a lack of surgeon experience, this objective is not always reached ${ }^{5-7}$ because optimal treatment may require extensive and complicated procedures in the upper abdomen such as liver or pancreatic resection. ${ }^{8}$ In those cases, when complete cytoreduction cannot be achieved, neoadjuvant chemotherapy treatment seems to be preferable, instead of primary debulking surgery. ${ }^{9}$ Moreover, biology and tumoral histology play an important role in the development 
and extension of the disease, and it has to be taken into account at the time of first treatment.

Splenic metastasis occurs rarely in malignant tumors. Based on studies of various tumors, the prevalence of splenic metastasis has been reported as ranging from $2.3 \%$ to $7.1 \%{ }^{10}$ The exact incidence of splenic metastasis in ovarian cancer is hard to determine. A study on 428 autopsies on patients with various histologic types of ovarian cancer noted splenic metastasis to be as high as $20 \% .{ }^{11}$ Usually, the disease does not invade the splenic parenchyma; however, the capsule of the spleen and the splenic flexure of the colon along with the gastrocolic ligament may be more commonly affected. Occasionally, it is necessary to remove an intact spleen to remove the whole tumoral mass. ${ }^{12-14}$ This procedure could increase the rate of intraoperative complications as well as postoperative morbidity. Most of the evidence comes from case reports and only few published studies. Because of that, neoadjuvant treatment could have a role to avoid splenectomy, although by the moment, no information has been published assessing that. Because of it, splenectomy still seems to be the best option to achieve the complete cytoreduction of the malignancy.

The objective of this study was to compare perioperative features and postoperative complications between patients who underwent splenectomy and those who did not at the time of primary cytoreductive surgery for advanced ovarian cancer. Secondarily, we compared oncological outcomes as well.

\section{MATERIALS AND METHODS}

After institutional review board approval, we retrospectively reviewed the charts of patients who underwent splenectomy with or without distal pancreatectomy at the time of primary cytoreductive surgery for advanced ovarian cancer (International Federation of Gynecology and Obstetrics [FIGO] stages IIIC-IV) ${ }^{15}$ at the European Institute of Oncology in Milan. From January 2001 to December 2008, among 259 patients with advanced ovarian cancers who underwent surgery, 33 cases were identified. Among all patients, we also selected 99 additional matched cases (ratio, 1:3) by age, American Society of Anesthesiology class, tumoral size (largest tumor bulk), presence of peritoneal carcinosis (defined as the presence of multiple tumoral implants on the peritoneal surfaces of the abdominal cavity, mostly in the upper abdomen), histology, nuclear grade, and surgical procedure but who did not undergo either splenectomy or pancreatectomy during the primary cytoreductive surgery for ovarian cancer.

Data collected included patients' age, all perioperative details including residual tumor as well as pathologic findings, FIGO stage, adjuvant therapy, and follow-up data.

Residual tumor was defined as maximal dimension of single largest cancer nodule at the end of cytoreductive surgery. Exclusion criteria included prior attempt of surgical cytoreduction at another institution, patients with stage IIIC disease based on nodal metastases alone, histology consistent with nonepithelial ovarian malignancies or borderline tumors, and neoadjuvant chemotherapy treatment.

Data collected were compared between the 2 groups (splenectomy vs no splenectomy). The statistical analysis was carried out by SPSS 15.0 software (SPSS Inc, Madrid, Spain). All statistical tests were two-sided, and differences were considered statistically significant at $P<0.05$. Disease-free survival was defined as the time interval from the date of surgery to the date of the documented first recurrence of disease. If there was no documented recurrence, disease-free survival was calculated from the date of surgery to the date of last follow-up or death, whichever occurred first. Overall survival was defined as the time interval from date of surgery to the date of death or last follow-up. The Kaplan-Meier method was used to estimate survival curves, and differences in survival were analyzed using the log-rank test.

\section{RESULTS}

Among the 132 records selected based on inclusion criteria, 33 patients $(25 \%)$ underwent splenectomy with or without distal pancreatectomy (case group) and 99 (75\%) did not (control group) at the time of cytoreductive surgery. Baseline

TABLE 1. Baseline characteristics of patients and malignancies

\begin{tabular}{|c|c|c|c|}
\hline Characteristic & $\begin{array}{l}\text { Case Group } \\
\quad \mathbf{n}=\mathbf{3 3}\end{array}$ & $\begin{array}{c}\text { Control Group } \\
\mathbf{n}=99\end{array}$ & $\boldsymbol{P}$ \\
\hline Age, mean $\pm \mathrm{SD}$ & $54.8 \pm 10.7$ & $55.2 \pm 9.7$ & ns \\
\hline ASA class, $\%$ & & & ns \\
\hline $\mathrm{I}$ & 6.1 & 7.1 & \\
\hline II & 27.2 & 40.8 & \\
\hline III & 66.7 & 52.1 & \\
\hline IV & 0 & 0 & \\
\hline $\begin{array}{l}\text { Size of largest mass, } \\
\text { mean } \pm \mathrm{SD}, \mathrm{cm}\end{array}$ & $20.3 \pm 10.7$ & $17.3 \pm 9.8$ & ns \\
\hline Final FIGO stage, $\%$ & & & $<0.01$ \\
\hline IIIC & 51.5 & 76.8 & \\
\hline IV & 48.5 & 23.2 & \\
\hline Peritoneal carcinosis, $\%$ & 90.9 & 85.9 & $\mathrm{~ns}$ \\
\hline $\begin{array}{l}\text { Ascites, mean } \pm \\
\text { SD, mL }\end{array}$ & $3205 \pm 2707$ & $2174 \pm 2297$ & 0.04 \\
\hline $\begin{array}{l}\text { Preoperative CA125, } \\
\text { mean } \pm \mathrm{SD}, \mathrm{UI} / \mathrm{mL}\end{array}$ & $3049 \pm 5194$ & $2959 \pm 6260$ & ns \\
\hline Histology, $\%$ & & & ns \\
\hline Clear cells & 6.1 & 1 & \\
\hline Endometrioid & 9.1 & 16.1 & \\
\hline Serous & 72.7 & 72.8 & \\
\hline Mixed & 12.1 & 10.1 & \\
\hline Nuclear grade, $\%$ & & & ns \\
\hline 1 & 3 & 4 & \\
\hline 2 & 24.2 & 14.2 & \\
\hline 3 & 63.6 & 79.8 & \\
\hline $\mathrm{N} / \mathrm{A}$ & 9.2 & 2 & \\
\hline
\end{tabular}

ASA, American Society of Anesthesiology; n, number of cases; N/A, not available; ns, not significant; SD, standard deviation. 
characteristics regarding patients and tumors of both groups are shown in Table 1. We observed significant differences between the groups with regard to final FIGO stage (48.5\% vs $23.2 \%$ stage IV in the case group compared to the controls, respectively; $P<0.01$ ). Among all FIGO stage IV cases, we observed $25 \%$ based on extra-abdominal lesions in the splenectomy group versus $39.1 \%$ in the control group. We also observed differences in the amount of ascites (3205 vs $2174 \mathrm{~mL}$, respectively; $P=0.04)$, although when we analyzed only the serous tumors, no statistical differences were found (2952 vs $2409 \mathrm{~mL}$, respectively; $P=0.35$ ).

Both groups were also comparable with respect to the surgical procedures performed. We did not observe any difference between the groups, with the exception, besides splenectomy, of distal pancreatectomy (Table 2). In the splenectomy group, 4 distal pancreatectomies (12.1\%) were carried out, whereas, as expected, none in the control group $(P<0.01)$. If we analyze only serous tumors, we also observe a significant higher rate of small bowel resection in the splenectomy group ( $12.5 \%$ vs $1.4 \%$, respectively; $P=0.01)$. Comparison of perioperative characteristics is shown in Table 3. We observed longer operating time in the splenectomy group (33 extra minutes in case group, $P=0.02$ ) as well as larger estimated blood loss (812 extra milliliters in the case group, $P=0.03$ ) and consequently intraoperative blood transfusion rate $(78.8 \%$ vs $42.4 \%$ in the case group compared to the controls, $P<0.01)$. The last 3 items seemed to be insignificant when we analyzed only serous carcinomas. Intensive care unit stay was also significantly longer in the splenectomy group $(1.4 \pm 1.3$ days) compared to that in the control group $(0.5 \pm$

TABLE 2. Comparison of surgical procedures by group

\begin{tabular}{lccc}
\hline Procedure & $\begin{array}{c}\text { Case Group } \\
\mathbf{n = 3 3 ,} \%\end{array}$ & $\begin{array}{c}\text { Control Group } \\
\mathbf{n =} \mathbf{9 9}, \boldsymbol{\%}\end{array}$ & $\boldsymbol{P}$ \\
\hline TAH/BSO & 97 & 100 & $\mathrm{~ns}$ \\
Rectosigmoid resection & 72.7 & 62.6 & $\mathrm{~ns}$ \\
Small bowel resection & 9.1 & 4 & $\mathrm{~ns}$ \\
Hemicolectomy L/R & 6.1 & 5 & $\mathrm{~ns}$ \\
Transverse colectomy & 6.1 & 4 & $\mathrm{~ns}$ \\
Ileocecal resection & 6.1 & 8.1 & $\mathrm{~ns}$ \\
Total omentectomy & 93.9 & 98 & $\mathrm{~ns}$ \\
Lymph nodes dissection & 39.4 & 45.5 & $\mathrm{~ns}$ \\
Diaphragmatic surgery & & & $\mathrm{ns}$ \\
$\quad$ Stripping & 45.5 & 41.4 & \\
$\quad$ Resection & 27.3 & 15.2 & \\
Distal pancreatectomy & 12.1 & 0 & $<0.01$ \\
Liver resection & 15.2 & 6.1 & $\mathrm{~ns}$ \\
Cholecystectomy & 3 & 2 & $\mathrm{~ns}$ \\
Gastric resection & 0 & 2 & $\mathrm{~ns}$ \\
Ileostomy & 3 & 0 & $\mathrm{~ns}$ \\
Colostomy & 0 & 1 & $\mathrm{~ns}$ \\
\hline
\end{tabular}

BSO, Bilateral salpingo-oophorectomy; L/R, left/right; TAH, total abdominal hysterectomy.
TABLE 3. Perioperative features

\begin{tabular}{lccc}
\hline & $\begin{array}{c}\text { Case } \\
\text { Group n = 33 } \\
\text { Characteristics }\end{array}$ & $\begin{array}{c}\text { Control } \\
\text { Group n = 99 }\end{array}$ & \\
\hline Mean RT, mm \pm SD) & (Mean \pm SD) & $\boldsymbol{P}$ \\
Residual tumor, mm & $7.1 \pm 10.5$ & $12.2 \pm 21.9$ & 0.07 \\
$\quad$ & & & $\mathrm{~ns}$ \\
$\quad 0.1-10$ & $32.4 \%$ & $42.4 \%$ & \\
$\quad>10$ & $18.2 \%$ & $37.4 \%$ & \\
Operating time, min & $305 \pm 62$ & $272 \pm 72$ & 0.02 \\
EBL, mL & $1828 \pm 1373$ & $1016 \pm 983$ & 0.03 \\
Intraoperative BT & $78.8 \%$ & $42.4 \%$ & $<0.01$ \\
Postoperative BT & $63.6 \%$ & $52.5 \%$ & $\mathrm{~ns}$ \\
Parenteral nutrition & $57.6 \%$ & $43.4 \%$ & $\mathrm{~ns}$ \\
ICU stay, days & $1.4 \pm 1.3$ & $0.5 \pm 0.8$ & $<0.01$ \\
Length of stay, days & $11.1 \pm 5.7$ & $9.5 \pm 3.3$ & $\mathrm{~ns}$ \\
\hline
\end{tabular}

BT, Blood transfusion; EBL, estimated blood loss; ICU, intensive care unit; RT, residual tumor.

0.8 days). Moreover, residual tumor (Table 3), although not statistically significant $(P=0.07)$, tends to be smaller in the splenectomy group (mean of $7.1 \mathrm{~mm}$ ) compared to the control group (mean of $12.2 \mathrm{~mm}$.). However, when we analyzed only serous tumors, we observed statistical significance (5.3 \pm $6.9 \mathrm{~mm}$ in the splenectomy group vs $13.8 \pm 23.9 \mathrm{~mm}$ in the controls; $P=0.008)$ and larger significant differences if we subanalyzed grade 3 tumors $(5.2 \pm 7.2 \mathrm{~mm}$ in the splenectomy group vs $16.1 \pm 25.7 \mathrm{~mm}$ in the controls; $P=0.007$ ).

We did not observe significant differences among most postoperative complications, with the exception of pleural effusion rate, which was higher $(P=0.02)$ in the splenectomy group, as well as the rate of postoperative pneumonia $(P=0.01)$ (Table 4$)$. When we selected grade 3 serous tumors, no significant differences were observed in pleural effusion rates (50\% in the splenectomy group vs $28.1 \%$ in the controls; $P=0.18)$. However, hospital stay was not significantly longer in the splenectomy group.

The mean \pm SD follow-up time was $32 \pm 21.5$ months (30.2 \pm 20.7 months in the splenectomy group and $32.4 \pm 21.9$ in the control group). The patients received a mean number of cycles of chemotherapy of $5.6 \pm 2.2$ in the splenectomy group vs $6.4 \pm 1.8$ cycles in the control group $(P=0.06)$. In the first group, $75.8 \%$ received carboplatin + paclitaxel and $9.1 \%$ carboplatin + docetaxel, whereas in the control group, the percentages were $74.7 \%$ and $8.1 \%$, respectively. The rest of the patients received other combinations of chemotherapy. At the time of follow-up, we observed $33.3 \%$ and $36.4 \%$ of patients alive with disease and dead of disease, respectively, in the splenectomy group compared to $26.3 \%$ and $40.4 \%$, respectively, in the control group $(P=0.74)$. No significant differences were found in disease free and overall survival rates, which were $30.3 \%$ and $66.6 \%$, respectively, in the splenectomy group and $33.3 \%$ and $59.6 \%$, respectively, in the control group. Moreover, no differences were observed also 
TABLE 4. Postoperative complications

\begin{tabular}{lccc}
\hline Complications & $\begin{array}{c}\text { Case Group } \\
\mathbf{n}=\mathbf{3 3}, \mathbf{n}(\mathbf{\%})\end{array}$ & $\begin{array}{c}\text { Control Group } \\
\mathbf{n}=\mathbf{9 9 ,} \mathbf{n}(\%)\end{array}$ & $\boldsymbol{P}$ \\
\hline Anastomotic leak & $1(3)$ & $2(2)$ & $\mathrm{ns}$ \\
Pelvic abscess & $0(0)$ & $2(2)$ & $\mathrm{ns}$ \\
UTI & $1(3)$ & $2(2)$ & $\mathrm{ns}$ \\
Wound infection & $3(9.1)$ & $5(5.1)$ & $\mathrm{ns}$ \\
Thromboembolism & $2(6.1)$ & $1(1)$ & $\mathrm{ns}$ \\
Pleural effusion & $15(45.5)$ & $19(19.2)$ & 0.02 \\
Pneumothorax & $3(9.1)$ & $6(6.1)$ & $\mathrm{ns}$ \\
Subphrenic abscess & $1(3)$ & $1(1)$ & $\mathrm{ns}$ \\
Pneumonia & $2(6.1)$ & $0(0)$ & 0.01 \\
Sepsis & $1(3)$ & $1(1)$ & $\mathrm{ns}$ \\
Others & & & \\
$\quad$ Cardiovascular & $3(9.1)$ & $5(5.1)$ & $\mathrm{ns}$ \\
$\quad$ Urinary & $1(3)$ & $3(3)$ & $\mathrm{ns}$ \\
Gastrointestinal & $1(3)$ & $7(7.1)$ & $\mathrm{ns}$ \\
$\quad$ Nerve injury & $1(3)$ & $2(2)$ & $\mathrm{ns}$ \\
\hline
\end{tabular}

UTI, Urinary tract infection.

in the time to relapse between groups $(13.8 \pm 10.9$ months in the splenectomy group vs $17.3 \pm 12.1$ months in the control group). Similarly, overall survival curves did not show significant differences $(P=0.48)$ between the groups with the $\log$ rank test as well (Fig. 1). Same results were observed after a subanalysis on serous tumors and grade 3 tumors.

\section{DISCUSSION}

Primary cytoreductive surgery is the cornerstone of the initial approach of patients with diagnosis of advanced ovarian cancer (FIGO stages II-IV); and because the goal of such surgery should be the reduction to microscopic disease of the whole malignancy, ${ }^{8}$ splenectomy should not be a limitation to achieve such an aim when involved by disease.

Isolated splenic parenchymal lesions are rare. A literature search performed through Pubmed yielded only reports of a few cases. ${ }^{10,16-19}$ Several studies have proposed different hypotheses to explain this phenomenon, most notably suggesting that the splenic capsule could act as a shield. The contractile properties of the spleen might also be involved, as well as local splenic immunomechanisms. A suggested poor development of afferent lymphatics to the spleen or perhaps the tortuosity of the splenic artery could also be responsible of the low involvement rate. Abdominal fluid circulation may also explain the lower rate of carcinomatosis in the left upper quadrant. Among isolated splenic metastasis, isolated splenic parenchymal lesions are more infrequent and represent the hematogenous spreading of the malignancy. ${ }^{10,17,18}$

Chen et al, ${ }^{20}$ in 2000 , evaluated the role of splenectomy at the time of surgical cytoreduction in advanced ovarian cancer and concluded that it could be performed with acceptable morbidity. More authors in more recent years suggest to incorporate upper abdominal resection in advanced ovarian cancer to achieve a complete cytoreduction. ${ }^{21,22}$

We compared 2 groups of patients who underwent primary cytoreductive surgery, matched by clinical characteristics and surgical procedures with the exception of splenectomy with or without distal pancreatectomy, to evaluate the role of spleen removal to achieve complete cytoreduction of perioperative characteristics and postoperative complications. To our knowledge, this is the first paper that presents a casematch design to address this issue.

The 2 groups were comparable; only FIGO stage IV and ascites volume were significantly higher in the splenectomy group, indirectly confirming the larger extent of disease in that group, although in further analysis for serous tumors, ascites seemed to be the same for both groups.

Concerning the perioperative features, we observed, as expected, longer operative time, larger estimated blood loss, and higher intraoperative blood transfusion rate in the splenectomy group; an extra day of intensive care unit stay (ICU) was also observed in the case group. The differences in operative time could be not clinically relevant, although we consider important the increase in blood loss and the stay in the ICU unit in patients' longer healing and higher hospital expenses. These differences could be related to the presence of a significant higher percentage of more advanced stage disease in this group. Slightly higher rates of procedures in the upper abdomen were observed in the splenectomy group. No complications associated to the 4 distal pancreatectomies performed in the group of splenectomy were observed. Despite of longer ICU stay for the splenectomy group, no significant differences were noted in total length of hospital stay.

The most common complications related to splenectomy reported in the literature are left-sided atelectasis, thromboembolic events, and pneumonia. ${ }^{23,24}$ Magtibay et $\mathrm{al}^{24}$ reported on 112 splenectomies (66 at primary cytoreduction for advanced ovarian cancer), and the perioperative morbidity and mortality rates were $15 \%$ and $5 \%$, respectively, with none of the deaths directly related to the splenectomy. ${ }^{25}$

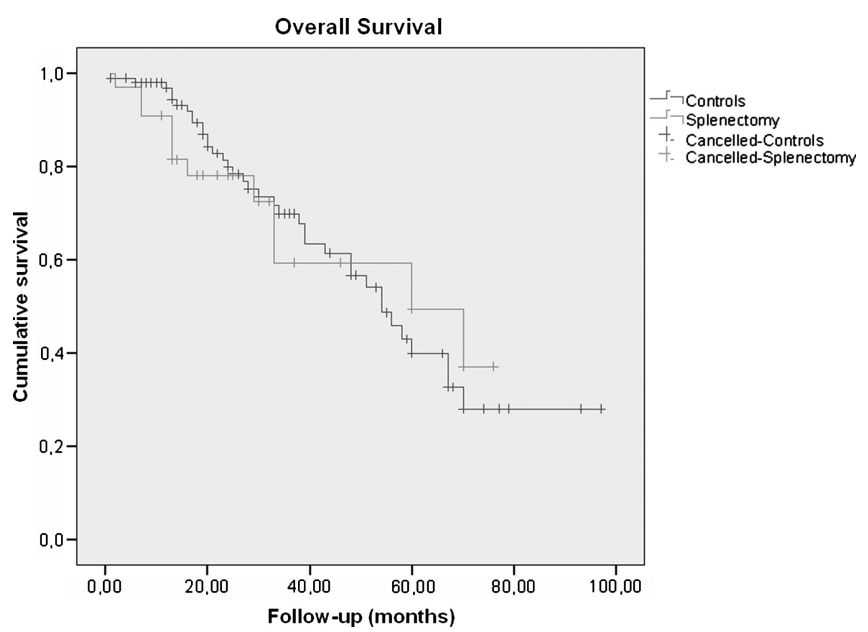

FIGURE 1. Comparison between splenectomy group and control group of overall survival curves calculated by Kaplan-Meier method $(P=0.48)$. 
Regarding postoperative complications, we did not observe significant differences between groups, with the exception of a significant higher pleural effusion and pneumonia rate observed in the splenectomy group. The former may be related to the extent of the disease in the upper abdomen, with a higher rate of diaphragm involvement. In fact, although the difference was not statistically significant between the groups, we observed a larger number of patients who underwent diaphragmatic stripping and resection in the splenectomy group compared to the controls. Moreover, the larger ascites volume in the splenectomy group (mean of 3205 vs $2174 \mathrm{~mL}$, respectively; $P=0.04$ ) supports the hypothesis that in this group, more disseminated disease could be present. We also observed $6.1 \%$ patients who developed postoperative pneumonia in the splenectomy group compared to $0 \%$ in the control, may be due to the absence of the spleen, which contains the largest reserve of the body's monocytes and, together with the activity of the reticuloendothelial system, promotes the tissue healing and avoids the infections by capsulate bacterias. ${ }^{26}$ Our results are very close to the data reported by Magtibay et al, ${ }^{24}$ who describe $4.4 \%$ of pneumonia and $4.4 \%$ of sepsis ( $3 \%$ in our case group). Similarly, Chi et $\mathrm{al}^{27}$ published on 45 splenectomies and described that a higher rate of pneumonia may be caused by the splenectomy; however, the removal of the involved spleen to achieve a complete primary cytoreduction increases the overall survival rate in these patients. 8,28

In the present study, the remaining postoperative complication rates are acceptable and similar in the 2 groups. Again, despite a higher rate of pleural effusion and pneumonia for the splenectomy group, no significant differences were noted in total length of hospital stay.

Moreover, residual tumor, although not statistically significant $(P=0.07)$, tends to be smaller in the splenectomy group compared to the control group; and probably with larger groups of patients, this difference could reach statistical significance. Moreover, after selection of serous and grade 3 tumors, significant differences were observed. This difference has a special interest, as cytoreduction to nodules less than $1 \mathrm{~cm}$ in maximum diameter seems to be associated with a clear benefit of overall survival compared to those cases with larger residual disease. ${ }^{8,28}$ In this study, we found same rates of residual tumor less than $1 \mathrm{~cm}: 81.1 \%$ in the splenectomy group versus $79.8 \%$ in the control group $(87.5 \%$ vs $78.8 \%$, respectively, for serous tumors).

In the current study, we did not observe differences between the groups in the disease-free survival and overall survival rates. We believe this represents a positive result of oncological outcomes, as the splenectomy group had a significant higher rate $(25.3 \%)$ of FIGO stage IV disease usually believed to have a poorer prognosis than stage IIIC. ${ }^{29}$ Therefore, the amount of residual disease at the end of primary cytoreductive surgery rather than initial bulk of tumor seems to be a more significant prognostic factor for oncology outcomes of these groups of patients.

Other studies observed that patients with ovarian cancer with splenic metastasis presented a poorer prognosis than those without splenic metastasis; but again, the difference was not statistically significant. ${ }^{30,31}$
In conclusion, splenectomy, with distal pancreatectomy if pancreatic tail is involved, at the time of primary cytoreductive surgery for patients with advanced ovarian cancer may contribute to achieve complete cytoreduction with acceptably low perioperative complication and mortality rates. Therefore, this procedure seems to be an acceptable and feasible intervention in the effort to achieve no residual disease and therefore to improve the oncologic outcomes of these patients.

\section{REFERENCES}

1. Jemal A, Siegel R, Xu J, et al. Cancer statistics, 2010. CA Cancer J Clin. 2010;60:277-300.

2. Ibeanu OA, Bristow RE. Predicting the outcome of cytoreductive surgery for advanced ovarian cancer: a review. Int J Gynecol Cancer. 2010;20:S1-S11.

3. Marchetti C, Pisano C, Facchini G, et al. First-line treatment of advanced ovarian cancer: current research and perspectives. Expert Rev Anticancer Ther. 2010;10:47-60.

4. Fader AN, Rose PG. Role of surgery in ovarian carcinoma. J Clin Oncol. 2007;25:2873-2883.

5. Bristow RE, Montz FJ. Complete surgical cytoreduction of advanced ovarian carcinoma using the argon beam coagulator. Gynecol Oncol. 2001;83:39-48.

6. Eisenkop SM, Spirtos NM, Friedman RL, et al. Relative influences of tumor volume before surgery and the cytoreductive outcome on survival for patients with advanced ovarian cancer: a prospective study. Gynecol Oncol. 2003;90:390-396.

7. Crawford SC, Vasey PA, Paul J, et al. Does aggressive surgery only benefit patients with less advanced ovarian cancer? Results from an international comparison within the SCOTROC-1 Trial. J Clin Oncol. 2005;23:8802-8811.

8. Shih KK, Chi DS. Maximal cytoreductive effort in epithelial ovarian cancer surgery. J Gynecol Oncol. 2010;21:75-80.

9. Vergote I, Tropé CG, Amant F, et al. Neoadjuvant chemotherapy or primary surgery in stage IIIC or IV ovarian cancer. N Engl J Med. 2010;363:943-953.

10. Koh YS, Kim JC, Cho CK. Splenectomy for solitary splenic metastasis of ovarian cancer. BMC Cancer. 2004;4:96.

11. Rose PG, Piver MS, Tsukada Y, et al. Metastatic patterns in histologic variants of ovarian cancer. An autopsy study. Cancer. 1989;64:1508-1513.

12. Deppe G, Zbella EA, Skogerson K, et al. The rare indication for splenectomy as part of cytoreductive surgery in ovarian cancer. Gynecol Oncol. 1983;16:282-287.

13. Sonnendecker EW, Guidozzi F, Margolius KA. Splenectomy during primary maximal cytoreductive surgery for epithelial ovarian cancer. Gynecol Oncol. 1989;35:301-306.

14. Guidozzi F, Ball JH. Extensive primary cytoreductive surgery for advanced epithelial ovarian cancer. Gynecol Oncol. 1994;53:326-330.

15. FIGO Annual Report. Int J Gynecol Obstet. 2006;105:3-4.

16. Manci N, Bellati F, Muzii L, et al. Splenectomy during secondary cytoreduction for ovarian cancer disease recurrence: surgical and survival data. Ann Surg Oncol. 2006;13: $1717-1723$.

17. Otrock ZK, Seoud MA, Khalifeh MJ, et al. Laparoscopic splenectomy for isolated parenchymal splenic metastasis of ovarian cancer. Int J Gynecol Cancer. 2006;16:1933-1935.

18. Alloni R, Garberini A, Caputo D, et al. Solitary splenic metastasis of ovarian carcinoma: report of two cases. Surg Today. 2008;38:1144-1147. 
19. Yoshioka R, Okabayashi T, Nishimori I, et al. A long-survived case with solitary splenic metastasis from ovarian carcinoma. Surg Technol Int. 2008;17:192-194.

20. Chen LM, Leuchter RS, Lagasse LD, et al. Splenectomy and surgical cytoreduction for ovarian cancer. Gynecol Oncol. 2000;77:362-368.

21. Kehoe SM, Eisenhauer EL, Chi DS. Upper abdominal surgical procedures: liver mobilization and diaphragm peritonectomy/ resection, splenectomy, and distal pancreatectomy. Gynecol Oncol. 2008;111:S51-S55.

22. Leblanc E, Narducci F, Boulanger L, et al. Upper abdomen cytoreduction in advanced ovarian carcinoma: techniques and results. Bull Cancer. 2009;96:1199-1205.

23. Nicklin JL, Copeland LJ, O'Toole RV, et al. Splenectomy as part of cytoreductive surgery for ovarian carcinoma. Gynecol Oncol. 1995;58:244-247.

24. Magtibay PM, Adams PB, Silverman MB, et al. Splenectomy as part of cytoreductive surgery in ovarian cancer. Gynecol Oncol. 2006;102:369-374.

25. Swirski FK, Nahrendorf M, Etzrodt M, et al. Identification of splenic reservoir monocytes and their deployment to inflammatory sites. Science. 2009;325:612-616.
26. Chi DS, Zivanovic O, Levinson KL, et al. The incidence of major complications after the performance of extensive upper abdominal surgical procedures during primary cytoreduction of advanced ovarian, tubal and peritoneal carcinomas. Gynecol Oncol. 2010;119:38-42.

27. Chi DS, Eisenhauer EL, Lang J, et al. What is the optimal goal of primary cytoreductive surgery for bulky stage IIIC epithelial ovarian carcinoma (EOC)? Gynecol Oncol. 2006;103: 559-564.

28. Zapardiel I, Morrow CP. New terminology for cytoreduction in advanced ovarian cancer. Lancet Oncol. 2011;12:214.

29. Heintz AP, Odicino F, Maisonneuve P, et al. Carcinoma of the ovary. FIGO 6th annual report on the results of treatment in gynecological cancer. Int J Gynaecol Obstet. 2006;95: S161-S192.

30. Ayhan A, Al RA, Baykal C, et al. The influence of splenic metastases on survival in FIGO stage IIIC epithelial ovarian cancer. Int J Gynecol Cancer. 2004;14:51-56.

31. Eisenkop SM, Spirtos NM, Lin WC. Splenectomy in the context of primary cytoreductive operations for advanced epithelial ovarian cancer. Gynecol Oncol. 2006;100:344-348. 